\title{
Preparation of cotton materials using CORONA discharge
}

Carneiro, N.; Souto, A.P.; Nogueira, C.; Madureira, A.; Krebs, C.; Cooper, S.

\begin{abstract}
The present work concerns the study of preparation processes when this operation is preceded by a CORONA discharge made on dry raw fabrics. The influence in the whiteness degree, hydrophility, starch removal and uniformity of properties of the cellulosic material after preparation is studied. Combined preparation processes are widely used in order to replace solutions where desizing, alkaline boiling and bleaching are individual operations, being a main interest to find the optimal conditions to achieve alkaline oxidative treatments with an uniform and more intense cleaning action. CORONA discharge is able to strongly modify hydrophility of cotton fabrics in raw stage, which is determinant to increase liquids absorption and uniformity of processed fabrics. Results of long-bath, pad-roll, pad-batch and pad-steam bleaching processes in laboratory conditions are compared using or not wetting agent in the recipes and including CORONA discharge as a preparation operation. The transfer of this technology to industry regarding the implementation in textile plant in a very near future is being worked, supported by results as the present ones.
\end{abstract}

